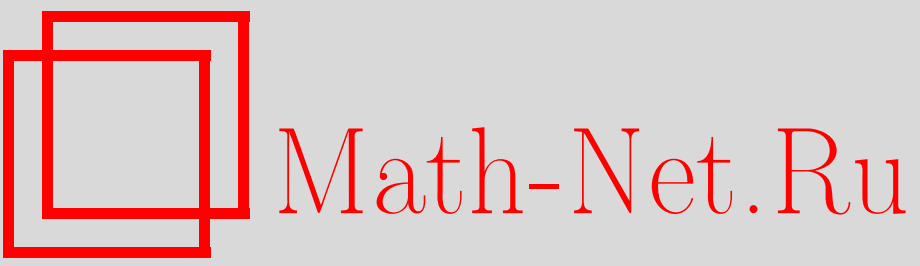

А. В. Шапошников, О сильной единственности решений вырожденных двумерных стохастических уравнений, Теория вероятн. и ее примен., 2011, том 56, выпуск 2, 301-317

DOI: https://doi.org/10.4213/tvp4376

Использование Общероссийского математического портала Math-Net.Ru подразумевает, что вы прочитали и согласны с пользовательским соглашением

http: //www . mathnet.ru/rus/agreement

Параметры загрузки:

IP : 44.207 .124 .84

26 апреля 2023 г., 16:20:26 
2011

(C) 2011 г

ШАПОШНИКОВ А.В.*

\title{
О СИЛЬНОЙ ЕДИНСТВЕННОСТИ РЕШЕНИЙ ВЫРОЖДЕННЫХ ДВУМЕРНЫХ СТОХАСТИЧЕСКИХ УРАВНЕНИЙ ${ }^{1)}$
}

\begin{abstract}
Доказано существование сильного решения и его потраекторная единственность для стохастических дифференциальных уравнений на плоскости с вырожденной матрицей диффузии и ограниченным борелевским сносом специального вида. Метод основан на оценках производных Маллявэна решений стохастических уравнений и критерии компактности в классе Соболева на винеровском пространстве.
\end{abstract}

Ключевые слова и фразыл: стохастичекое дифференциальное уравнение, сильное решение, потраекторная единственность, производная Маллявэна, критерий компактности.

В данной работе рассматриваются стохастические дифференциальные уравнения (СДУ) в $\mathbf{R}^{2}$ следующего вида:

$$
\begin{cases}d X_{t}=Y_{t} d t & X_{0}=x_{0} \\ d Y_{t}=b\left(X_{t}, Y_{t}\right) d t+d W_{t}, & Y_{0}=y_{0}\end{cases}
$$

Некоторые эргодические свойства систем СДУ этого типа (наличие инвариантной меры, перемешивание, сходимость к стационарному режиму) рассматривались в работах [1]-[3], в которых также обсуждались вопросы существования и единственности решений уравнений указанного вида. В работе [1] доказано, что если снос $b$ является борелевской функцией и растет не более чем линейно, т.е. $|b(x, y)| \leqslant C(1+|x|+|y|)$, то рассматриваемое уравнение имеет слабое решение; при этом решение единственно в смысле распределения и является строго марковским процессом. В работе [3] доказано существование сильного решения при некоторых дополнительных предположениях (липшицевости или монотонности по переменной $y$ сноса $b$ ). В статье [1] ставится вопрос о существовании сильного решения в случае борелевской функции $b$. Основной результат данной заметки состоит в следующей теореме.

\footnotetext{
* Московский государственный университет им. М.В.Ломоносова, механикоматематический факультет, Ленинские горы, 119991 Москва, Россия.

1) Работа поддержана грантом РФФИ № 10-01-00518 и программой SFB 701 при университете Билефельда.
} 
Теорема 1. Для стохастического дифференииального уравнения

$$
\begin{cases}d X_{t}=Y_{t} d t & X_{0}=x_{0}, \\ d Y_{t}=b\left(\alpha X_{t}+\beta Y_{t}\right) d t+d W_{t}, & Y_{0}=y_{0},\end{cases}
$$

где $\alpha, \beta \in \mathbf{R}, \beta \neq 0, b$ - ограниченная борелевская функиия, справедливь следующие утверждения:

(i) при указанных условиях сильное решение существует;

(ii) для решений данного уравнения выполняется потраекторная единственность.

Д о к а з а т е л ь с т в о. Вначале опишем общий план построения сильного решения.

1) Рассматриваются последовательность уравнений со «сглаженными» коэффициентами $\left\{b_{n}\right\}$, которые в подходящем смысле сходятся к функции $b$, и последовательность решений этих уравнений $\left\{\left(X^{n}, Y^{n}\right)\right\}$. Далее, используя дифференцируемость в смысле Маллявэна решений уравнений с гладкими коэффициентами, мы доказываем, что найдется такое $\delta>0$, что для каждого $t \in[0, \delta]$ последовательности $\left\{X_{t}^{n}\right\}$ и $\left\{Y_{t}^{n}\right\}$ образуют относительно компактные множества в пространстве $L^{2}(\mu)$.

2) Следующий шаг состоит в том, чтобы доказать, что найдутся такие подпоследовательность $\left\{n_{k}\right\}$ и прогрессивно измеримый относительно фильтрации $\left(\mathscr{F}_{t}^{W}\right)_{t \geqslant 0}$ процесс $\left(X^{0}, Y^{0}\right)$, что последовательность $\left\{\left(X^{n_{k}}, Y^{n_{k}}\right)\right\}$ сходится к процессу $\left(X^{0}, Y^{0}\right)$ в $L^{2}([0, \delta] \times C[0,1], \mathscr{B} \mathscr{F}, \lambda \otimes \mu)$, где $\mathscr{B} \mathscr{F}$ обозначает $\sigma$-алгебру прогрессивно измеримых относительно потока $\left(\mathscr{F}_{t}^{W}\right)_{t \geqslant 0}$ множеств.

3) Следующий этап заключается в доказательстве того, что найдется согласованный с потоком $\left(\mathscr{F}_{t}^{W}\right)_{t \geqslant 0}$ процесс $(\widehat{X}, \widehat{Y})$ с почти наверное непрерывными траекториями, который является решением уравнения (1) на отрезке $[0, \delta]$.

4) Далее, используя марковское свойство слабого решения, мы доказываем, что сильное решение существует на всем временном промежутке $[0, \infty)$.

5) Наконец, утверждение (ii) вытекает из утверждения (i) и известной теоремы о том, как связаны между собой потраекторная единственность, сильное существование и слабая единственность (см. [4]).

3 а м е ч а н и е 1. В работе [5] применяется идейно близкая схема построения сильного решения в случае единичной матрицы диффузии и ограниченного борелевского сноса. Основные отличия заключаются в различных подходах к исследованию уравнения для производной в смысле Маллявэна решения СДУ с гладким коэффициентом сноса. При получении необходимых оценок производных решений СДУ в работе [5] на с. 3937 используется явное представление (формула (48)) решения линейного неоднородного ОДУ со «случайными» коэффициентами. Поскольку в общем случае матрицы, задающие данное дифференциальное 
уравнение, не обязаны коммутировать, то, по-видимому, приведенное в [5] рассуждение в полной общности охватывает только одномерный случай.

Перейдем к реализации намеченного плана. Заметим, что достаточно рассмотреть случай, когда $\beta=1$, поскольку общая ситуация сводится к данной с помощью введения новой функции $\widehat{b}(x)=b(\beta x)$. Итак, далее будем считать, что $\beta=1$. Пусть $\left\{b_{n}\right\}-$ последовательность финитных бесконечно дифференцируемых функций, почти всюду по мере Лебега сходящаяся к функции $b$ и удовлетворяющая условию $\left\|b_{n}\right\|_{\infty} \leqslant\|b\|_{\infty}$. Пусть $d-$ некоторое положительное число. Обозначим через $W$ пространство $C_{0}[0, d]$ непрерывных функций $w$ на отрезке $[0, d]$ таких, что $w(0)=0$, наделенное стандартной sup-нормой $\|\cdot\|_{\infty}$. Пусть $\mu$ - стандартная мера Винера на борелевской $\sigma$-алгебре пространства $W$. Через $H$ обозначим гильбертово пространство $W_{0}^{1,2}[0, d]$, состоящее из абсолютно непрерывных функций $h$ на отрезке $[0, d]$, которые удовлетворяют следующим условиям:

$$
h(0)=0, \quad\|h\|_{H}^{2}<\infty, \quad \text { где } \quad\|h\|_{H}^{2}=\int_{[0, d]}\left|h^{\prime}(t)\right|^{2} d t .
$$

Напомним, что пространство $H$ является пространством КамеронаМартина меры $\mu$. Также, когда это будет удобно, будем обозначать меру Винера через $\mathbb{P}$ и считать, что она задана на $C[0, \infty)$. Напомним определение класса Соболева $\mathbb{D}^{1,2}$ на пространстве $W$ с мерой Винера $\mu$. Пусть $\mathscr{F} \mathscr{C}_{b}^{\infty}$ обозначает совокупность всех функций $f$ на $W$ вида

$$
f(x)=\varphi\left(l_{1}(x), \ldots, l_{n}(x)\right), \quad \varphi \in C_{b}^{\infty}\left(\mathbf{R}^{n}\right), \quad l_{i} \in W^{*}, \quad n \in \mathbf{N} .
$$

Пространство Соболева $\mathbb{D}^{1,2}$ определяется (см. [6], см. также [7], [8]) как пополнение $\mathscr{F} \mathscr{C}_{b}^{\infty}$ относительно нормы $\|f\|_{1,2}=\|f\|_{L^{2}(\mu)}+\|\nabla f\|_{L^{2}(\mu, H)}$, где $\nabla f$ обозначает градиент функции $f$ вдоль $H$. Для фиксированной функции $F$ из пространства $\mathbb{D}^{1,2}$ отображение $\nabla F: W \rightarrow H$ можно рассматривать как вещественнозначную функцию двух аргументов $(w, t)$, где $w \in W, t \in[0, d]$, причем при фиксированном $w$ эта функция принадлежит классу $W_{0}^{1,2}[0, d]$ по переменной $t$. Следуя [6], обозначим производную этой функции по $t$ (при фиксированном $w$ ) в точке $s \in[0, d]$ через $D_{s} F(w)$. Таким образом, если $i$ - естественный изоморфизм $H$ и $L^{2}[0, d]$, переводящий $h$ в $h^{\prime}$, то элемент $i(\nabla F(w))$ пространства $L^{2}[0, d]$ есть функция $s \mapsto D_{t} F(w)$.

Доказательство относительной компактности последовательностей $\left\{X_{t}^{n}\right\}$ и $\left\{Y_{t}^{n}\right\}$ основано на следующих двух утверждениях (см. [9]).

Предложение 1. Пусть $C$ - компактный самосопряженный оператор на $H$ с плотным образом. Тогда для каждого с $>0$ множество

$$
\mathfrak{M}=\left\{F \in \mathbb{D}^{1,2}(W): \int_{W}|F|^{2} d \mu+\int_{W}\left\|C^{-1}(\nabla F)\right\|^{2} d \mu \leqslant c\right\}
$$


относительно компактно в $L^{2}(\mu)$.

Доказательство предложения 1 можно найти также в книге [7] (см. гл. $5, \S 12$ ).

Предложение 2. Для любого $\gamma>0$ единичный шар $B$ относительно нормьи

$$
\|f\|_{L^{2}[0,1]}+\left(\int_{0}^{1} \int_{0}^{1} \frac{|f(t)-f(s)|^{2}}{|t-s|^{1+\gamma}} d t d s\right)^{1 / 2}
$$

компактен в топологии пространства $L^{2}[0,1]$. Следовательно, найдется такой инъективный компактный самосопряженньий оператор $S$ в $L^{2}[0,1]$, что образ единичного шара при действии $S$ содержит $B$.

3 а м е ч а н и е 2. Приведенная норма эквивалентна норме пространства Бесова $B_{2,2}^{\gamma / 2}[0,1]$ (см. [10]), доказательство компактности в $L^{2}[0,1]$ единичного шара относительно указанной нормы также можно найти в [10] (см. гл. 6, п. 26.3.3). Для полноты изложения приведем простое доказательство предложения 2 , используя критерий компактности в $L^{2}[0,1]$. Основной этап заключается в проверке равномерной непрерывности в $L^{2}$ указанного семейства функций. Из формулы замены переменных мы получаем следующее равенство:

$$
J:=\int_{0}^{1} \int_{0}^{1} \frac{|f(t)-f(s)|^{2}}{|t-s|^{1+\gamma}} d t d s=2 \int_{0}^{1} \frac{1}{h^{1+\gamma}} \int_{0}^{1-h}|f(s+h)-f(s)|^{2} d s d h .
$$

Положим

$$
\varphi_{1}(h)=\int_{0}^{1-2 h}|f(s+h)-f(s)|^{2} d s, \quad \varphi_{2}(h)=\int_{0}^{1-h}|f(s+h)-f(s)|^{2} d s .
$$

Тогда для любого $h \in(0,1 / 2)$ и любого $u \in(0, h)$ выполняется неравенство $\varphi_{1}(h) \leqslant 2 \varphi_{1}(u)+2 \varphi_{2}(h-u)$. Временно зафиксируем $h \in(0,1 / 2)$ и проинтегрируем обе части этого неравенства по $u$ по интервалу $(0, h)$ :

$$
\begin{aligned}
h \varphi_{1}(h) & \leqslant 2 \int_{0}^{h} \varphi_{1}(u) d u+2 \int_{0}^{h} \varphi_{2}(h-u) d u \\
& \leqslant 2 h^{1+\gamma} \int_{0}^{h} \frac{\varphi_{1}(u)}{u^{1+\gamma}} d u+2 h^{1+\gamma} \int_{0}^{h} \frac{\varphi_{2}(h-u)}{(h-u)^{1+\gamma}} d u \\
& \leqslant 4 h^{1+\gamma} \int_{0}^{1} \frac{1}{v^{1+\gamma}} \int_{0}^{1-v}|f(s+v)-f(s)|^{2} d s d v .
\end{aligned}
$$

Следовательно, выполняется неравенство $\varphi_{1}(h) \leqslant 4 h^{\gamma} J$. Заменив $f$ на функцию, равную $f(1-x)$, можно получить аналогичную оценку и для

$$
\psi_{1}(h)=\int_{2 h}^{1}|f(s-h)-f(s)|^{2} d s
$$

Дальнейшее обоснование несложно. 
Следствие 1. Для любых $c, \gamma>0$ множество

$$
\mathfrak{N}=\left\{F \in \mathbb{D}^{1,2}(W): \mathbb{E}|F|^{2}+\mathbb{E} \int_{0}^{d} \int_{0}^{d} \frac{\left|D_{t} F-D_{s} F\right|^{2}}{|t-s|^{1+\gamma}} d t d s \leqslant c\right\}
$$

относительно компактно в $L^{2}(\mu)$.

Д о к а з а т е л ь с т в о. Пусть $i$ обозначает естественный изоморфизм пространств $H$ и $L^{2}[0, d]$, который каждому вектору $h \in H$ ставит в соответствие функцию $h^{\prime} \in L^{2}[0, d]$. Определим оператор $C$ в пространстве $H$ следующей формулой: $C=i^{-1} \circ S \circ i$, где $S-$ компактный самосопряженный оператор из предложения 2. Для завершения доказательства осталось воспользоваться предложением 1.

Основная цель дальнейших рассуждений заключается в том, чтобы получить такие оценки производных от решений уравнений со сглаженными коэффициентами $b_{n}$, которые бы позволили воспользоваться следствием 1.

Теперь временно предположим, что $b-$ финитная бесконечно дифференцируемая функция. Пусть $(X, Y)$ - решение соответствующего уравнения, где $X$ обозначает функцию двух переменных $(t, w) \mapsto X_{t}(w)$. Как известно, в этом случае $X_{t}(w), Y_{t}(w)$ бесконечно дифференцируемы в смысле Маллявэна (см. [8], [11]), причем для фиксированной функции $h \in H$ производная

$$
V_{t}=\left(\begin{array}{c}
\partial_{h} X_{t} \\
\partial_{h} Y_{t}
\end{array}\right)
$$

будет удовлетворять следующему уравнению со «случайными» коэффициентами:

$$
d V_{t}=\left(\begin{array}{cc}
0 & 1 \\
\alpha b^{\prime}\left(\alpha X_{t}+Y_{t}\right) & b^{\prime}\left(\alpha X_{t}+Y_{t}\right)
\end{array}\right) V_{t} d t+\left(\begin{array}{c}
0 \\
h^{\prime}(t)
\end{array}\right) d t, \quad V_{0}=\left(\begin{array}{l}
0 \\
0
\end{array}\right) .
$$

Рассмотрим также вспомогательное однородное матричное уравнение

$$
d M_{t}=\left(\begin{array}{cc}
0 & 1 \\
\alpha b^{\prime}\left(\alpha X_{t}+Y_{t}\right) & b^{\prime}\left(\alpha X_{t}+Y_{t}\right)
\end{array}\right) M_{t} d t, \quad M_{0}=\left(\begin{array}{ll}
1 & 0 \\
0 & 1
\end{array}\right),
$$

причем его решение будем обозначать через

$$
M_{t}=\left(\begin{array}{cc}
f_{1}(t, \alpha X+Y) & f_{2}(t, \alpha X+Y) \\
g_{1}(t, \alpha X+Y) & g_{2}(t, \alpha X+Y)
\end{array}\right)
$$

3 а м е ч а н и е 3 . В случае гладкого коэффициента $b$ мы можем считать, что для всех пар $\left(x_{0}, y_{0}\right)$ выбраны такие версии процессов $\left(X_{t}, Y_{t}\right)$, что для каждого $t$ случайные величины $X_{t}^{\left(x_{0}, y_{0}\right)}$ и $Y_{t}^{\left(x_{0}, y_{0}\right)}$ гладко 
зависят от начальных условий $\left(x_{0}, y_{0}\right)$. Тогда для каждого $t$ коэффициенты матрицы $M_{t}$ совпадают с производными по начальным условиям от $\left(X_{t}, Y_{t}\right)$, т.е.

$$
M_{t}=\left(\begin{array}{ll}
\frac{\partial X_{t}}{\partial x_{0}} & \frac{\partial X_{t}}{\partial y_{0}} \\
\frac{\partial Y_{t}}{\partial x_{0}} & \frac{\partial Y_{t}}{\partial y_{0}}
\end{array}\right) .
$$

Нетрудно убедиться, что $V_{t}$ можно представить в виде

$$
V_{t}=M_{t} \int_{0}^{t} M_{s}^{-1}\left(\begin{array}{c}
0 \\
h^{\prime}(s)
\end{array}\right) d s=\int_{0}^{t} M_{t} M_{s}^{-1}\left(\begin{array}{c}
0 \\
h^{\prime}(s)
\end{array}\right) d s .
$$

Положим $q(t)=b^{\prime}\left(\alpha X_{t}+Y_{t}\right)$. Уравнения для нахождения первого и второго столбцов матрицы $M_{t}$ отличаются лишь начальными условиями и имеют вид $f^{\prime}(t)=g(t), f^{\prime \prime}(t)=\alpha q(t) f(t)+q(t) g(t), f(0)=f_{0}, g(0)=g_{0}$. Далее нам также понадобится функция $r(t)$, которая определяется из равенства

$$
g(t)=\exp \left(\int_{0}^{t} q(s) d s\right) r(t)
$$

Нетрудно заметить, что в этом случае (с учетом предыдущего уравнения) справедливо равенство

$$
r^{\prime}(t)=\alpha q(t) \exp \left(-\int_{0}^{t} q(s) d s\right) f(t) .
$$

Применяя формулу интегрирования по частям, получаем

$$
\begin{aligned}
r(t) & =g_{0}+\int_{0}^{t}\left(-\alpha \exp \left(-\int_{0}^{s} q(l) d l\right)\right)^{\prime} f(s) d s \\
& =g_{0}-\alpha \exp \left(-\int_{0}^{t} q(s) d s\right) f(t)+\alpha f_{0}+\int_{0}^{t} \alpha r(s) d s \\
& =g_{0}+\alpha f_{0}-\alpha \exp \left(-\int_{0}^{t} q(s) d s\right)\left(f_{0}+\int_{0}^{t} g(s) d s\right)+\int_{0}^{t} \alpha r(s) d s .
\end{aligned}
$$

Умножая полученное равенство на $\exp \left(\int_{0}^{t} q(s) d s\right)$, мы получаем, что

$$
\begin{aligned}
g(t)= & \left(g_{0}+\alpha f_{0}\right) \exp \left(\int_{0}^{t} q(s) d s\right)-\alpha f_{0}-\alpha \int_{0}^{t} g(s) d s \\
& +\alpha \int_{0}^{t} g(s) \exp \left(\int_{s}^{t} q(l) d l\right) d s .
\end{aligned}
$$

Напомним, что нас интересуют те два случая, когда набор $\left(f_{0}, g_{0}\right)$ равен $(1,0)$ или $(0,1)$, поэтому далее мы будем предполагать, что выполняется одно из этих равенств. Тогда для каждого $p \geqslant 1$ существует такое 
$C_{0}=C_{0}(p)$, что для любого $t \in[0,1]$ справедливо неравенство, которое и будет лежать в основе существенной части дальнейших рассуждений:

$$
\begin{aligned}
|g(t)|^{p} \leqslant C_{0}( & \exp \left(p \int_{0}^{t} q(s) d s\right)+1+\int_{0}^{t}|g(s)|^{p} d s \\
& \left.+\int_{0}^{t}|g(s)|^{p} \exp \left(p \int_{s}^{t} q(l) d l\right) d s\right) .
\end{aligned}
$$

Заметим, что в случае $b \in C_{0}^{\infty}(\mathbf{R})$ (который мы сейчас и рассматриваем) решение уравнения (3) может быть получено как предел последовательных приближений на каждом отрезке, длина которого не превосходит $\delta_{0}\left(\left\|b^{\prime}\right\|_{\infty}\right)$, независимо от начальных условий в левом конце отрезка. Из этого замечания нетрудно получить, что $f(s)$ и $g(s)$ как функции от $w$ (винеровской траектории) при каждом фиксированном $s$ измеримы относительно $\mathscr{F}_{s}^{W}$, при этом найдется число $C\left(\left\|b^{\prime}\right\|_{\infty}\right)$ такое, что $\sup _{s \in[0,1]}(|f(s)|+|g(s)|) \leqslant C\left(\left\|b^{\prime}\right\|_{\infty}\right)$ для всякого $w$. Ниже нам понадобится такое наблюдение (которое также вытекает из последнего замечания): если $b \in C_{0}^{\infty}(\mathbf{R})$, то $f(s)$ и $g(s)$ зависят от $w$ только «через» процесс $Z=\alpha X+Y$, т.е. для каждого $s \in[0,1]$ мы имеем $f(s)=f(s, Z)$ и $g(s)=g(s, Z)$, где в данном случае $Z$ обозначает траекторию процесса $Z$ до момента $s$ включительно.

Чтобы оценить $\mathbb{E}|F(Z)|^{p}$, воспользуемся тем, что решение уравнения (1) может быть получено с помощью аналога теоремы Гирсанова (см. [1]), и тем, что имеет место единственность распределения для решений данного уравнения (см. [1]). Итак, если мы рассмотрим процессы $\widetilde{Y}_{t}=y_{0}+W_{t}, \widetilde{X}_{t}=x_{0}+\int_{0}^{t} \widetilde{Y}_{s} d s$, то относительно меры

$$
\varrho d \mathbb{P}=\exp \left(\int_{0}^{1} b\left(\alpha \widetilde{X}_{t}+\tilde{Y}_{t}\right) d W_{t}-\frac{1}{2} \int_{0}^{1} b^{2}\left(\alpha \widetilde{X}_{t}+\tilde{Y}_{t}\right) d t\right) d \mathbb{P}
$$

пара $\left(\tilde{X}_{t}, \tilde{Y}_{t}\right)$ будет решением рассматриваемого СДУ (вместе с некоторым новым винеровским процессом). Тогда мы получаем, что

$$
\mathbb{E}|F(Z)|^{p}=\int|F(\alpha \tilde{X}+\tilde{Y})|^{p} \varrho d \mathbb{P} \leqslant\left(\int|F(\alpha \tilde{X}+\tilde{Y})|^{2 p} d \mathbb{P}\right)^{1 / 2}\left(\int \varrho^{2 p} d \mathbb{P}\right)^{1 / 2},
$$

причем из теоремы Гирсанова следует, что выполняется неравенство

$$
\int \varrho^{2 p} d \mathbb{P} \leqslant C_{2}\left(\|b\|_{\infty}\right)<\infty
$$

Положим

$$
\varrho_{0}=\exp \left(\int_{0}^{d} \tilde{Y}_{t} d W_{t}-\frac{1}{2} \int_{0}^{d} \tilde{Y}_{t}^{2} d t\right) .
$$


Тогда справедливо следующее неравенство:

$$
\begin{aligned}
\int|F(\alpha \tilde{X}+\tilde{Y})|^{2 p} d \mathbb{P} & =\int|F(\alpha \tilde{X}+\tilde{Y})|^{2 p} \varrho_{0}^{1 / 2} \varrho_{0}^{-1 / 2} d \mathbb{P} \\
& \leqslant\left(\int|F(\alpha \tilde{X}+\tilde{Y})|^{4 p} \varrho_{0} d \mathbb{P}\right)^{1 / 2}\left(\int \varrho_{0}^{-1} d \mathbb{P}\right)^{1 / 2} .
\end{aligned}
$$

Используя распределение максимума винеровского процесса, нетрудно получить, что можно так выбрать значение $d$, не зависящее от $x_{0}, y_{0}, p$ и $b$, чтобы выполнялось неравенство

$$
\int \varrho_{0}^{-2} d \mathbb{P}+\int \varrho_{0}^{2} d \mathbb{P}<\infty
$$

Поскольку относительно меры $\varrho_{0} d \mathbb{P}$ процесс $\alpha \tilde{X}+\tilde{Y}$ имеет вид $\alpha x_{0}+y_{0}+$ $\widetilde{W}$, где $\widetilde{W}-$ винеровский процесс относительно меры $\rho d \mathbb{P}$, то в итоге мы получаем, что существует константа $C_{3}\left(\|b\|_{\infty}, x_{0}, y_{0}\right)$ такая, что

$$
\mathbb{E}|F(\alpha X+Y)|^{p} \leqslant C_{3}\left[\mathbb{E}\left|F\left(\alpha x_{0}+y_{0}+W\right)\right|^{4 p}\right]^{1 / 4} .
$$

Для сокращения записи будем считать, что $x_{0}=0$ и $y_{0}=0$. Пусть $R(x)=2 \int_{0}^{x} b(z) d z$. Тогда функция $R$ является липшицевой с константой Липшица $2\|b\|_{\infty}$, при этом $R$ бесконечно дифференцируема, если $b \in$ $C_{0}^{\infty}(\mathbf{R})$. Применяя формулу Ито к функции $R$ и винеровскому процессу, находим, что

$$
R\left(W_{t}\right)=\int_{0}^{t} 2 b\left(W_{s}\right) d W_{s}+\int_{0}^{t} b^{\prime}\left(W_{s}\right) d s .
$$

С помощью данного равенства и теоремы Гирсанова мы можем получить следующую оценку, которая нам понадобится в дальнейшем:

$$
\begin{aligned}
& \mathbb{E}\left[\exp \left(\int_{s}^{t} b^{\prime}\left(W_{l}\right) d l\right) \mid \mathscr{F}_{s}^{W}\right] \leqslant \mathbb{E}\left[\exp \left(4\|b\|_{\infty}\left|W_{t}-W_{s}\right|\right) \mid \mathscr{F}_{s}^{W}\right] \\
& \quad+\mathbb{E}\left[\exp \left(8 \int_{s}^{t} b\left(W_{l}\right) d W_{l}-\frac{64}{2} \int_{s}^{t} b^{2}\left(W_{l}\right) d l\right) \mid \mathscr{F}_{s}^{W}\right] \\
& \quad+\mathbb{E}\left[\exp \left(\frac{64}{2} \int_{s}^{t} b^{2}\left(W_{l}\right) d l\right) \mid \mathscr{F}_{s}^{W}\right] \leqslant C_{4}\left(\|b\|_{\infty}\right) .
\end{aligned}
$$

Теперь, используя полученные выше вспомогательные неравенства, оценим $\mathbb{E}|g(t, W)|^{p}$. Из неравенства для $|g(t, W)|^{p}$, переходя к математическим ожиданиям и применяя теорему Фубини, мы получаем

$$
\begin{aligned}
\mathbb{E}|g(t, W)|^{p} \leqslant C_{0}( & C_{4}\left(p\|b\|_{\infty}\right)+1+\int_{0}^{t} \mathbb{E}|g(s, W)|^{p} d s \\
& \left.+\int_{0}^{t} \mathbb{E}\left[|g(s, W)|^{p} \exp \left(p \int_{s}^{t} b^{\prime}\left(W_{l}\right) d l\right)\right] d s\right)
\end{aligned}
$$




$$
\begin{aligned}
=C_{0}( & C_{4}\left(p\|b\|_{\infty}\right)+1+\int_{0}^{t} \mathbb{E}|g(s, W)|^{p} d s \\
& \left.\quad+\int_{0}^{t} \mathbb{E}\left[|g(s, W)|^{p} \mathbb{E}\left[\exp \left(p \int_{s}^{t} b^{\prime}\left(W_{l}\right) d l\right) \mid \mathscr{F}_{s}^{W}\right]\right] d s\right) \\
\leqslant & C_{0}\left(C_{4}\left(p\|b\|_{\infty}\right)+1+\int_{0}^{t}\left(1+C_{4}\left(p\|b\|_{\infty}\right)\right) \mathbb{E}|g(s, W)|^{p} d s\right) .
\end{aligned}
$$

Из неравенства Гронуолла следует, что существует такая константа $C_{5}\left(p,\|b\|_{\infty}, \alpha\right)$, что для каждого $t \in[0, d]$ выполняется неравенство $\mathbb{E}|g(s, W)|^{p} \leqslant C_{5}\left(p,\|b\|_{\infty}, \alpha\right)$.

Следующий шаг состоит в том, чтобы доказать, что для каждого $p_{0} \geqslant 1$ существуют положительные числа $d\left(\|b\|_{\infty}, p_{0}\right)$ и $C_{6}\left(p_{0},\|b\|_{\infty}, \alpha\right)$ со следующим свойством: для любого $p \in\left[1, p_{0}\right]$ и любой пары чисел $s, t \in[0, d]$ справедливо неравенство $\mathbb{E}|g(t, W)-g(s, W)|^{p} \leqslant C_{6}|t-s|^{1 / 4}$. Для этого нам потребуется следующее вспомогательное утверждение.

Лемма 1. Для каждого $p_{0} \geqslant 1$ существуют иисла $d\left(\|b\|_{\infty}, p_{0}\right) u$ $C_{7}\left(p_{0},\|b\|_{\infty}, \alpha\right)>0$ такие, что для всякого $p \in\left[1, p_{0}\right]$ и всяких чисел $s, t \in[0, d]$ выполняется неравенство

$$
\mathbb{E}\left|\exp \left(\int_{s}^{t} b^{\prime}\left(W_{l}\right) d l\right)-1\right|^{p} \leqslant C_{7}|t-s|^{1 / 2} .
$$

Д о к а з а т е л ь с т в о. Заметим, что из теоремы Гирсанова и неравенства Дуба можно получить следующую оценку, которая выполняется при $p \in\left[1, p_{0}\right]$ и $s, t \in[0,1]$ :

$$
\begin{aligned}
\mathbb{E} & {\left[\max _{\theta \in[s, t]} \exp \left(\int_{s}^{\theta} 8 p b\left(W_{l}\right) d W_{l}\right)\right] } \\
= & \mathbb{E}\left[\max _{\theta \in[s, t]} \exp \left(\int_{s}^{\theta} 8 p b\left(W_{l}\right) d W_{l}-\int_{s}^{\theta} 64 p^{2} b^{2}\left(W_{l}\right) d l+\int_{s}^{\theta} 64 p^{2} b^{2}\left(W_{l}\right) d l\right)\right] \\
\leqslant & \mathbb{E}\left[\max _{\theta \in[s, t]} \exp \left(\int_{s}^{\theta} 16 p b\left(W_{l}\right) d W_{l}-\frac{1}{2} \int_{s}^{\theta} 256 p^{2} b^{2}\left(W_{l}\right) d l\right)\right] \\
& +\mathbb{E}\left[\max _{\theta \in[s, t]} \exp \left(\int_{s}^{\theta} 128 p^{2} b^{2}\left(W_{l}\right) d l\right)\right] \leqslant C_{8}\left(p,\|b\|_{\infty}, \alpha\right) .
\end{aligned}
$$

Пользуясь явной формулой для плотности распределения максимума винеровского процесса, мы можем получить, что найдется такое число $d\left(\|b\|_{\infty}, p_{0}\right)>0$, для которого имеет место оценка

$$
\mathbb{E}\left[\exp \left(8 p_{0}\|b\|_{\infty} \max _{\theta \in[s, t]}\left|W_{\theta}-W_{s}\right|\right)\right]<C_{9}\left(p_{0},\|b\|_{\infty}\right) .
$$

Применим следующее неравенство Буркхольдера-Дэвиса-Ганди для мартингалов (см., например, [11]): $\mathbb{E}\left(\max _{s \in[0, t]}\left|M_{s}\right|^{2 p}\right) \leqslant C(p) \mathbb{E}\langle M\rangle_{t}^{p}$, где 
$\langle M\rangle$ обозначает квадратическую вариацию мартингала $M$. Оно показывает, что для всех чисел $p$, лежащих в отрезке $\left[1, p_{0}\right]$, будут выполняться неравенства

$$
\mathbb{E}\left|\int_{s}^{t} 2 b\left(W_{l}\right) d W_{l}\right|^{2 p} \leqslant C_{10}\left(p_{0}\right) \mathbb{E}\left|\int_{s}^{t} 4 b^{2}\left(W_{l}\right) d l\right|^{p} \leqslant C_{10}\left(p_{0}\right) \cdot 4^{p}\|b\|_{\infty}^{2 p}|t-s| .
$$

Из доказанных выше оценок и цепочки неравенств

$$
\begin{aligned}
\mathbb{E} \mid & \exp \left(\int_{s}^{t} b^{\prime}\left(W_{l}\right) d l\right)-\left.1\right|^{p} \leqslant \mathbb{E}\left[\max _{\theta \in[s, t]} \exp \left(p \int_{s}^{\theta} b^{\prime}\left(W_{l}\right) d l\right)\left|\int_{s}^{t} b^{\prime}\left(W_{l}\right) d l\right|^{p}\right] \\
\leqslant & \left.\mathbb{E}\left[\max _{\theta \in[s, t]} \exp \left(2 p \int_{s}^{\theta} b^{\prime}\left(W_{l}\right) d l\right)\right]\right)^{1 / 2}\left(\mathbb{E}\left|\int_{s}^{t} b^{\prime}\left(W_{l}\right) d l\right|^{2 p}\right)^{1 / 2} \\
\leqslant & C_{11}\left(p,\|b\|_{\infty}, \alpha\right)\left(\mathbb{E}\left[\exp \left(8 p\|b\|_{\infty} \max _{\theta \in[s, t]}\left|W_{\theta}-W_{s}\right|\right)\right]\right. \\
& \left.+\mathbb{E}\left[\max _{\theta \in[s, t]} \exp \left(\int_{s}^{\theta} 8 p b\left(W_{l}\right) d W_{l}\right)\right]\right)^{1 / 2} \\
& \times\left(\mathbb{E}\left(2\|b\|_{\infty}\left|w_{t}-w_{s}\right|\right)^{2 p}+\mathbb{E}\left|\int_{s}^{t} 2 b\left(W_{l}\right) d W_{l}\right|^{2 p}\right)^{1 / 2} \\
\leqslant & C_{7}\left(p_{0},\|b\|_{\infty}, \alpha\right)|t-s|^{1 / 2}
\end{aligned}
$$

следует утверждение леммы.

Наконец, используя интегральное равенство для $g$, мы получаем, что если $t$ и $s$ лежат в отрезке $\left[0, d\left(\|b\|_{\infty}, p_{0}\right)\right]$ и $p \in\left[1, p_{0} / 8\right]$ (где $d\left(\|b\|_{\infty}, p_{0}\right)$ - то же, что и в лемме), то выполняется неравенство $\mathbb{E}|g(t, W)-g(s, W)|^{p} \leqslant(12+12|\alpha|)^{p}\left(\Delta_{1}+\Delta_{2}+\Delta_{3}\right)$, где

$$
\begin{aligned}
\Delta_{1}= & \mathbb{E}\left[\exp \left(\int_{0}^{s} p b^{\prime}\left(W_{u}\right) d u\right)\left|\exp \left(\int_{s}^{t} b^{\prime}\left(W_{l}\right) d l\right)-1\right|^{p}\right] \\
\leqslant & \left(\mathbb{E} \exp \left(\int_{0}^{s} 2 p b^{\prime}\left(W_{u}\right) d u\right)\right)^{1 / 2}\left(\mathbb{E}\left|\exp \left(\int_{s}^{t} b^{\prime}\left(W_{l}\right) d l\right)-1\right|^{2 p}\right)^{1 / 2} \\
\leqslant & C_{12}\left(p_{0},\|b\|_{\infty}, \alpha\right)|t-s|^{1 / 4}, \\
\Delta_{2}= & \mathbb{E}\left|\int_{s}^{t} g(u, W) d u\right|^{p} \leqslant|t-s|^{p / 2} \mathbb{E} \int_{0}^{1}\left(1+|g(u, W)|^{2 p}\right) d u \\
\leqslant & |t-s|^{p / 2}\left(1+\int_{s}^{t} \mathbb{E}|g(u, W)|^{2 p} d u\right) \\
\leqslant & |t-s|^{p / 2}\left(1+C_{5}\right) \leqslant|t-s|^{1 / 2}\left(1+C_{5}\right) \\
\Delta_{3}= & \mathbb{E} \mid \int_{0}^{t} g(u, W) \exp \left(\int_{u}^{t} b^{\prime}\left(W_{l}\right) d l\right) d u \\
& \quad-\left.\int_{0}^{s} g(u, W) \exp \left(\int_{u}^{s} b^{\prime}\left(W_{l}\right) d l\right) d u\right|^{p} \\
\leqslant & 2^{p}\left(\mathbb{E}\left|\int_{0}^{s} g(u, W) \exp \left(\int_{u}^{s} b^{\prime}\left(W_{l}\right) d l\right)\left(\exp \left(\int_{s}^{t} b^{\prime}\left(W_{l}\right) d l\right)-1\right) d u\right|^{p}\right.
\end{aligned}
$$




$$
\begin{aligned}
& \left.+\mathbb{E}\left|\int_{s}^{t} g(u, W) \exp \left(\int_{u}^{t} b^{\prime}\left(W_{l}\right) d l\right) d u\right|^{p}\right) \\
& \leqslant C_{13}\left(p_{0},\|b\|_{\infty}, \alpha\right)|t-s|^{1 / 4} .
\end{aligned}
$$

Поскольку $f^{\prime}=g$, то из оценки (6) для $\mathbb{E}|g(t, W)|^{p}$ мы получаем, что

$$
\begin{gathered}
\mathbb{E}|f(t, W)-f(s, W)|^{p} \leqslant C_{14}\left(p_{0},\|b\|_{\infty}, \alpha\right)|t-s|^{1 / 2} \\
\times \mathbb{E}\left(1+\int_{s}^{t}|g(u, W)|^{2 p} d u\right) \leqslant C_{15}\left(p_{0},\|b\|_{\infty}, \alpha\right)|t-s|^{1 / 2}, \\
\mathbb{E}|f(t, W)|^{p} \leqslant C_{16}\left(p_{0},\|b\|_{\infty}, \alpha\right) .
\end{gathered}
$$

Временно зафиксируем $a \in\left[0, d\left(\|b\|_{\infty}, p_{0}\right)\right]$. Пусть $k_{i, j}(s, \alpha X+Y)$ обозначают коэффициенты матрицы $M_{a} M_{s}^{-1}$, т.е.

$$
M_{a} M_{s}^{-1}=\left(\begin{array}{ll}
k_{1,1}(s, \alpha X+Y) & k_{1,2}(s, \alpha X+Y) \\
k_{2,1}(s, \alpha X+Y) & k_{2,2}(s, \alpha X+Y)
\end{array}\right)
$$

Докажем, что для каждого $p_{0} \geqslant 1$ найдется такая константа $C_{17}\left(p_{0},\|b\|_{\infty}, \alpha\right)$, что для всякого $p \in\left[1, p_{0}\right]$ и всякой пары точек $s, t \in$ $\left[0, d\left(\|b\|_{\infty}, p_{0}\right)\right]$ справедливы неравенства

$$
\mathbb{E}\left|k_{i, j}(t, W)-k_{i, j}(s, W)\right|^{p} \leqslant C_{17}|t-s|^{1 / 16}, \quad \mathbb{E}\left|k_{i, j}(s, W)\right|^{p} \leqslant C_{17} .
$$

Доказательство основано на двух приводимых ниже предложениях.

Предложение 3. Если для случайньх функиий $\varphi$ и величинь $\mathbb{E}|\varphi(t)-\varphi(s)|^{2 p}, \mathbb{E}|\psi(t)-\psi(s)|^{2 p}, \mathbb{E}|\varphi(s)|^{2 p}, \mathbb{E}|\psi(s)|^{2 p}$ конечныл, то справедливо неравенство

$$
\begin{aligned}
\mathbb{E}|\varphi(t) \psi(t)-\varphi(s) \psi(s)|^{p} \leqslant & 2^{p}\left(\mathbb{E}|\varphi(t)|^{2 p}\right)^{1 / 2}\left(\mathbb{E}|\psi(t)-\psi(s)|^{2 p}\right)^{1 / 2} \\
& +2^{p}\left(\mathbb{E}|\psi(s)|^{2 p}\right)^{1 / 2}\left(\mathbb{E}|\varphi(t)-\varphi(s)|^{2 p}\right)^{1 / 2} .
\end{aligned}
$$

Д о к а з а т е л ь с т в о. Обоснование несложно - надо воспользоваться оценкой $\mathbb{E}|\varphi(t) \psi(t)-\varphi(s) \psi(s)|^{p} \leqslant 2^{p} \mathbb{E}|\varphi(t) \psi(t)-\varphi(t) \psi(s)|^{p}+$ $2^{p} \mathbb{E}|\varphi(t) \psi(s)-\varphi(s) \psi(s)|^{p}$.

Предложение 4. Выполнено равенство

$$
\operatorname{det} M_{s}(W)=\exp \left(\int_{0}^{s} b^{\prime}\left(W_{l}\right) d l\right) .
$$

Д о к аз а т е ль с т в. Данное равенство вытекает непосредственно из формулы Лиувилля для определителя фундаментальной матрицы решений линейного обыкновенного дифференциального уравнения. 
Используя явную формулу для коэффициентов обратной матрицы, полученные выше оценки для $\left\{f_{i}\right\}$ и $\left\{g_{i}\right\}$, предложения 3,4 и лемму 1 , нетрудно получить требуемое утверждение для функций $\left\{k_{i, j}\right\}$. Далее, воспользовавшись неравенством (5), мы получаем, что для каждого $p_{0} \geqslant 1$ найдется константа $C_{18}\left(p_{0},\|b\|_{\infty}, \alpha\right)$, для которой выполняется следующее условие: для всякого $p \in\left[1, p_{0}\right]$ и всякой пары точек $s, t \in\left[0, d\left(\|b\|_{\infty}, p_{0}\right)\right]$ справедливы неравенства

$$
\begin{aligned}
& \mathbb{E}\left|k_{i, j}(t, \alpha X+Y)-k_{i, j}(s, \alpha X+Y)\right|^{p} \leqslant C_{18}|t-s|^{1 / 64}, \\
& \mathbb{E}\left|k_{i, j}(s, \alpha X+Y)\right|^{p} \leqslant C_{18} .
\end{aligned}
$$

Выше мы получили выражение (формула (4)) для производной вдоль вектора $h$ от функционалов $X_{a}(w), Y_{a}(w)$ :

$$
\left(\begin{array}{c}
\partial_{h} X_{a} \\
\partial_{h} Y_{a}
\end{array}\right)=\int_{0}^{a} M_{a} M_{s}^{-1}\left(\begin{array}{c}
0 \\
h^{\prime}(s)
\end{array}\right) d s
$$

Следовательно, справедливы следующие равенства:

$$
\begin{aligned}
D_{s} X_{a} & =I_{(0, a)}(s)\left(k_{1,1}(s, \alpha X+Y)+k_{1,2}(s, \alpha X+Y)\right), \\
D_{s} Y_{a} & =I_{(0, a)}(s)\left(k_{2,1}(s, \alpha X+Y)+k_{2,2}(s, \alpha X+Y)\right) .
\end{aligned}
$$

Поскольку

$$
\begin{aligned}
\mathbb{E} \int_{0}^{a} \int_{0}^{a} \frac{\left|k_{i, j}(t)-k_{i, j}(s)\right|^{2}}{|t-s|^{1+1 / 64}} d t d s & =\int_{0}^{a} \int_{0}^{a} \frac{\mathbb{E}\left|k_{i, j}(t)-k_{i, j}(s)\right|^{2}}{|t-s|^{1+1 / 64}} d t d s \leqslant C_{18} a^{2}, \\
\mathbb{E} \int_{0}^{a} k_{i, j}(s)^{2} d s & =\int_{0}^{a} \mathbb{E}\left(k_{i, j}(s)^{2}\right) d s \leqslant C_{18} a,
\end{aligned}
$$

то из следствия 1 мы получаем, что семейства функционалов $\left\{X_{a}^{n}\right\},\left\{Y_{a}^{n}\right\}$ $(a \in[0, d])$ образуют относительно компактные множества в $L^{2}(\mu)$.

Применяя диагональную процедуру, мы можем выбрать такую подпоследовательность $\left\{n_{k}\right\}_{k=1}^{\infty}$, что для каждого $t \in \mathbf{Q} \cap[0, d]$ последовательность $\left(X_{t}^{n_{k}}, Y_{t}^{n_{k}}\right)$ будет сходиться к некоторой паре элементов $\left(X_{t}^{00}, Y_{t}^{00}\right)$ из $L^{2}(\mu)$. Нетрудно заметить, что для этой подпоследовательности сходимость $\left(X_{t}^{n_{k}}, Y_{t}^{n_{k}}\right)$ в $L^{2}(\mu)$ будет иметь место не только для рациональных $t$, а вообще для всех вещественных $t \in[0, d]$. Это вытекает из следующих неравенств, которые выполняются для решений уравнений вида (1):

$$
\begin{aligned}
& \mathbb{E}\left|Y_{t}-Y_{s}\right|^{2} \leqslant 2 \mathbb{E}\left(\int_{s}^{t} b\left(\alpha X_{s}+Y_{s}\right) d s\right)^{2}+2 \mathbb{E}\left|W_{t}-W_{s}\right|^{2} \\
& \leqslant 2\|b\|_{\infty}^{2}(t-s)^{2}+2|t-s| \\
& \mathbb{E}\left|X_{t}-X_{s}\right|^{2}=\mathbb{E}\left|\int_{s}^{t} Y_{u} d u\right|^{2} \leqslant|t-s| \int_{s}^{t} \mathbb{E}\left|Y_{u}\right|^{2} d u
\end{aligned}
$$


Из приведенных выше оценок также вытекает, что последовательность двумерных процессов $\left(X^{n_{k}}, Y^{n_{k}}\right)$ сходится в $L^{2}([0, d] \times C[0,1], \mathscr{B} \mathscr{F}, \lambda \otimes$ $\mu)$, где $\mathscr{B} \mathscr{F}$ обозначает сигма-алгебру прогрессивно измеримых относительно потока $\left(\mathscr{F}_{t}^{W}\right)_{t \geqslant 0}$ множеств; при этом мы можем считать, что для почти всех по мере $\lambda \otimes \mu$ пар $(t, w)$ имеет место равенство $\lim _{k \rightarrow \infty}\left(X_{t}^{n_{k}}, Y_{t}^{n_{k}}\right)[w]=\left(X_{t}^{0}, Y_{t}^{0}\right)[w]$, где $\left(X^{0}, Y^{0}\right)$ - «предельный» прогрессивно измеримый процесс. Поскольку для каждого $t \in[0, d]$ имеет место сходимость $\left(X_{t}^{n_{k}}, Y_{t}^{n_{k}}\right)$ в $L^{2}(\mu)$, то для почти всех $t$ почти наверное справедливо равенство $\left(X_{t}^{00}, Y_{t}^{00}\right)=\left(X_{t}^{0}, Y_{t}^{0}\right)$. Теперь докажем, что для $\lambda$-почти всех $t$ из отрезка $[0, d]$ для пары процессов $\left(X^{0}, Y^{0}\right)$ почти наверное выполняются соотношения

$$
X_{t}^{0}=x_{0}+\int_{0}^{t} Y_{s}^{0} d s, \quad Y_{t}^{0}=y_{0}+\int_{0}^{t} b\left(\alpha X_{s}^{0}+Y_{s}^{0}\right) d s+W_{t} .
$$

Рассмотрим $t \in[0, d]$, для которого $\left\{\left(X_{t}^{n_{k}}, Y_{t}^{n_{k}}\right)\right\}$ сходится в среднем квадратическом к $\left(X_{t}^{0}, Y_{t}^{0}\right)$. Для упрощения обозначений будем считать, что выбранная подпоследовательность $\left\{n_{k}\right\}_{k=1}^{\infty}$ совпадает с исходной последовательностью $\{1,2,3, \ldots\}$. Поскольку при каждом $n$ пара процессов $\left(X^{n}, Y^{n}\right)$ является решением уравнения (1) со сносом $b_{n}$, то справедливы следующие равенства:

$$
X_{t}^{n}=x_{0}+\int_{0}^{t} Y_{s}^{n} d s, \quad Y_{t}^{n}=y_{0}+\int_{0}^{t} b_{n}\left(\alpha X_{s}^{n}+Y_{s}^{n}\right) d s+W_{t} .
$$

Для доказательства равенств (9) оценим сумму квадратов $L^{2}$-норм разностей правых и левых частей соотношений (9) и (10). В первом случае указанные нормы оцениваются с помощью выражения $2\left(\mathbb{E}\left|X_{t}^{n}-X_{t}^{0}\right|^{2}+\right.$ $\left.\mathbb{E} \int_{0}^{t}\left|Y_{t}^{n}-Y_{t}^{0}\right|^{2} d t\right)$, которое стремится к 0 , когда $n$ стремится к бесконечности. Для оценки квадрата $L^{2}$-нормы разности

$$
\int_{0}^{t} b_{n}\left(\alpha X_{s}^{n}+Y_{s}^{n}\right) d s-\int_{0}^{t} b\left(\alpha X_{s}^{0}+Y_{s}^{0}\right) d s
$$

нам потребуется еще одно вспомогательное утверждение.

Лемма 2. Для каждого $t \in[0, d]$, для которого имеет место сходимость $\left\{\left(X_{t}^{n}, Y_{t}^{n}\right)\right\} \kappa\left(X_{t}^{0}, Y_{t}^{0}\right)$ в $L^{2}(\mu)$, распределение вектора $\left(X_{t}^{0}, Y_{t}^{0}\right)$ совпадает с распределением вектора $\left(\widetilde{X}_{t}, \widetilde{Y}_{t}\right)$, где $\widetilde{Y}_{t}=y_{0}+W_{t}, \widetilde{X}_{t}=$ $x_{0}+\int_{0}^{t} \widetilde{Y}_{s} d s$, относительно мерь

$$
\varrho d \mathbb{P}=\exp \left(\int_{0}^{d} b\left(\alpha \tilde{X}_{t}+\tilde{Y}_{t}\right) d W_{t}-\frac{1}{2} \int_{0}^{d} b^{2}\left(\alpha \tilde{X}_{t}+\tilde{Y}_{t}\right) d t\right) d \mathbb{P} .
$$

Д о к а з а т е л ь с т в о. Заметим, что если в утверждении леммы заменить $b$ на $b_{n}$ и соответственно $\left(X_{t}^{0}, Y_{t}^{0}\right)$ на $\left(X_{t}^{n}, Y_{t}^{n}\right)$, а $\left(\tilde{X}_{t}, \widetilde{Y}_{t}\right)$ на 
$\left(\widetilde{X}_{t}^{n}, \widetilde{Y}_{t}^{n}\right)$, то аналогичное равенство будет выполняться в силу уже упоминавшегося ранее утверждения о слабых решениях рассматриваемого уравнения (которые можно построить с помощью теоремы Гирсанова). Теперь заметим, что так как по предположению векторы $\left\{\left(X_{t}^{n}, Y_{t}^{n}\right)\right\}$ сходятся к $\left(X_{t}^{0}, Y_{t}^{0}\right)$ в $L^{2}(\mu)$, то их распределения слабо сходятся к распределению $\left(X_{t}^{0}, Y_{t}^{0}\right)$. Заметим, что из сходимости последовательности $\left\{\varrho_{n}\right\}$ к $\varrho$ в $L^{1}(\mathbb{P})$ вытекает слабая сходимость распределений векторов $\left(\widetilde{X}_{t}^{n}, \widetilde{Y}_{t}^{n}\right)$ к распределению $\left(\widetilde{X}_{t}, \widetilde{Y}_{t}\right)$ (в этом нетрудно убедиться, используя определение слабой сходимости и теорему о замене переменных в интеграле Лебега). Напомним, что по построению $\left\{b_{n}\right\}$ - последовательность функций из $C_{0}^{\infty}(\mathbf{R})$, которая почти всюду по мере Лебега сходится к функции $b$, и при этом выполняется условие $\left\|b_{n}\right\|_{\infty} \leqslant\|b\|_{\infty}$. Тогда по теореме Лебега об ограниченной сходимости мы получаем, что последовательность процессов $b_{n}\left(\alpha \widetilde{X}_{t}+\widetilde{Y}_{t}\right)$ сходится в $L^{2}([0, d] \times C[0,1], \mathscr{B} \mathscr{F}, \lambda \otimes \mu)$ к процессу $b\left(\alpha \widetilde{X}_{t}+\widetilde{Y}_{t}\right)$, откуда нетрудно получить, что последовательность $\left\{\varrho_{n}\right\}$ сходится к $\varrho$ по вероятности. Наконец, поскольку найдется такое $C_{18}\left(d,\|b\|_{\infty}\right)$, что $\mathbb{E} \varrho_{n}^{2} \leqslant C_{18}$, то мы получаем, что последовательность $\left\{\varrho_{n}\right\}$ равномерно интегрируема, откуда с учетом полученной сходимости по вероятности и вытекает требуемое утверждение.

Теперь докажем, что $L^{2}$-норма разности (11) стремится к нулю, когда $n$ стремится к бесконечности. Зафиксируем число $\eta \in(0,1)$. В силу доказанной леммы и теоремы о мажорируемой сходимости можно найти номер $m_{0}(\eta)$ такой, что для каждого $m \geqslant m_{0}(\eta)$ выполняется неравенство

$$
\Lambda_{1}(m)=\mathbb{E} \int_{0}^{t}\left|b\left(\alpha X_{s}^{0}+Y_{s}^{0}\right)-b_{m}\left(\alpha X_{s}^{0}+Y_{s}^{0}\right)\right|^{2} d s \leqslant \eta .
$$

Так как последовательность $\left\{\left(X_{t}^{n}, Y_{t}^{n}\right)\right\}$ сходится по вероятности к $\left(X_{t}^{0}, Y_{t}^{0}\right)$ не только для данного $t$, но и для почти всех $s \in[0, d]$, то $\Lambda_{2}(m, n)$ стремится к нулю, когда $n$ стремится бесконечности, где

$$
\Lambda_{2}(m, n)=\mathbb{E} \int_{0}^{t}\left|b_{m}\left(\alpha X_{s}^{0}+Y_{s}^{0}\right)-b_{m}\left(\alpha X_{s}^{n}+Y_{s}^{n}\right)\right|^{2} d s .
$$

Наконец, при $n \geqslant m_{0}(\eta)$ оценим величину

$$
\Lambda_{3}(m, n)=\mathbb{E} \int_{0}^{t}\left|b_{m}\left(\alpha X_{s}^{n}+Y_{s}^{n}\right)-b_{n}\left(\alpha X_{s}^{n}+Y_{s}^{n}\right)\right|^{2} d s .
$$

Для этого снова воспользуемся теоремой Фубини и неравенством (5):

$$
\begin{aligned}
& \mathbb{E} \int_{0}^{t}\left|b_{m}\left(\alpha X_{s}^{n}+Y_{s}^{n}\right)-b_{n}\left(\alpha X_{s}^{n}+Y_{s}^{n}\right)\right|^{2} d s \\
& \quad=\int_{0}^{t} \mathbb{E}\left|b_{m}\left(\alpha X_{s}^{n}+Y_{s}^{n}\right)-b_{n}\left(\alpha X_{s}^{n}+Y_{s}^{n}\right)\right|^{2} d s
\end{aligned}
$$




$$
\begin{gathered}
\leqslant C_{3} \int_{0}^{t}\left(\mathbb{E}\left|b_{m}\left(\alpha x_{0}+y_{0}+W_{s}\right)-b_{n}\left(\alpha x_{0}+y_{0}+W_{s}\right)\right|^{8}\right)^{1 / 4} d s \\
\leqslant C_{3} \int_{\eta}^{t}\left(\mathbb{P}\left\{\left|b_{m}\left(\alpha x_{0}+y_{0}+W_{s}\right)-b_{n}\left(\alpha x_{0}+y_{0}+W_{s}\right)\right| \geqslant \eta\right\}\right. \\
\left.\times\left(2\|b\|_{\infty}\right)^{8}+\eta^{8}\right)^{1 / 4} d s+C_{3}(2\|b\|)^{2} \eta .
\end{gathered}
$$

Так как последовательность $\left\{b_{n}\right\}$ сходится почти всюду относительно меры Лебега, то с учетом полученного неравенства (при необходимости увеличивая $\left.m_{0}(\eta)\right)$ мы можем считать, что выполняется неравенство

$$
\mathbb{E} \int_{0}^{t}\left|b_{m}\left(\alpha X_{s}^{n}+Y_{s}^{n}\right)-b_{n}\left(\alpha X_{s}^{n}+Y_{s}^{n}\right)\right|^{2} d s \leqslant C_{3}(4+4\|b\|)^{2} \eta .
$$

Поскольку искомая разность (11) оценивается числом $8\left(\Lambda_{1}\left(m_{0}(\eta)\right)+\right.$ $\left.\Lambda_{2}\left(m_{0}(\eta), n\right)+\Lambda_{3}\left(m_{0}(\eta), n\right)\right)$, то мы получаем требуемое утверждение.

Определим прогрессивно измеримый относительно фильтрации $\left(\mathscr{F}_{t}^{W}\right)_{t \geqslant 0}$ процесс $\left(\widehat{X}_{t}, \widehat{Y}_{t}\right)$ на отрезке $[0, d]$ следующим образом:

$$
\widehat{X}_{t}=x_{0}+\int_{0}^{t} Y_{s}^{0} d s, \quad \widehat{Y}_{t}=y_{0}+\int_{0}^{t} b\left(\alpha X_{s}^{0}+Y_{s}^{0}\right) d s+W_{t} .
$$

В силу сказанного выше мы получаем, что для почти всех точек $t$ с вероятностью единица справедливо равенство $\left(\widehat{X}_{t}, \widehat{Y}_{t}\right)=\left(X_{t}^{0}, Y_{t}^{0}\right)$. Следовательно, для почти всех $t$ почти наверное выполняются равенства

$$
\widehat{X}_{t}=x_{0}+\int_{0}^{t} \widehat{Y}_{s} d s, \quad \widehat{Y}_{t}=y_{0}+\int_{0}^{t} b\left(\alpha \widehat{X}_{s}+\widehat{Y}_{s}\right) d s+W_{t},
$$

а так как в (13) обе части представляют собой процессы с почти наверное непрерывными траекториями, то на множестве полной меры эти процессы совпадают. Итак, мы построили сильное решение уравнения (1) на отрезке $\left[0, d\left(\|b\|_{\infty}\right)\right]$.

3 а м е ч а н и е 4. Из неравенств (7) и (8) нетрудно получить, что процесс $\left(X_{t}^{00}, Y_{t}^{00}\right)$ стохастически непрерывен, поэтому для каждого $t \in[0, d]$ почти наверное выполняется равенство $\left(X_{t}^{00}, Y_{t}^{00}\right)=\left(\widehat{X}_{t}, \widehat{Y}_{t}\right)$. Так как $X_{t}^{00}$ и $Y_{t}^{00}$ являются пределами в $L^{2}(\mu)$ последовательностей функционалов, ограниченных в совокупности по норме пространства $\mathbb{D}^{1,2}(W)$, то функционалы $\widehat{X}_{t}$ и $\widehat{Y}_{t}$ также принадлежат $\mathbb{D}^{1,2}(W)$.

Теперь докажем, что сильное решение существует на всем временном промежутке $[0, \infty)$. Пусть $\left(\widetilde{X}_{t}, \widetilde{Y}_{t}\right)-$ слабое решение уравнения (1) на некотором вероятностном пространстве. В работе [1] доказано, что в этом случае $\left(\widetilde{X}_{t}, \widetilde{Y}_{t}\right)$ будет однородным и строго марковским, при этом указано, что данное утверждение является следствием слабой единственности. Используя этот факт и теорему 3.1 из [4], мы получаем, что утверждение о единственности будет выполняться и для трехмерного 
процесса $\left(\widetilde{X}_{t}, \widetilde{Y}_{t}, \widetilde{W}_{t}\right)$, следовательно, он будет однородным и строго марковским (однако нам потребуется лишь однородность и марковость). Поскольку сильное решение $\left(\widehat{X}_{t}, \widehat{Y}_{t}\right)$ на отрезке $[0, d]$ было получено как предел решений уравнений с гладкими коэффициентами, то найдутся заданные на $\mathbf{R} \times \mathbf{R} \times[0, d] \times C[0, \infty)$ борелевские отображения (прогрессивно измеримые по переменным $t$ и $w$ ) - которые мы будем для удобства обозначать $X^{000}\left(x_{0}, y_{0}, t, w\right)$ и $Y^{000}\left(x_{0}, y_{0}, t, w\right)-$ со следующим свойством: для почти всех относительно меры $\lambda \otimes \lambda \otimes \lambda \otimes \mu$ наборов аргументов выполнено равенство

$$
\left(\widehat{X}_{t}, \widehat{Y}_{t}\right)=\left(X^{000}\left(x_{0}, y_{0}, t, w\right), Y^{000}\left(x_{0}, y_{0}, t, w\right)\right) .
$$

Определим «вспомогательный» процесс $\left(\widehat{X}_{t}^{0}, \widehat{Y}_{t}^{0}\right)$ для $t \in[d, 2 d]$ следующим образом:

$$
\begin{aligned}
\left(\widehat{X}_{t}^{0}, \widehat{Y}_{t}^{0}\right)= & \left(X^{000}\left(\widehat{X}_{d}, \widehat{Y}_{d}, t-d, W_{d \leqslant \leqslant 2 d}-W_{d}\right),\right. \\
& \left.Y^{000}\left(\widehat{X}_{d}, \widehat{Y}_{d}, t-d, W_{d \leqslant \leqslant 2 d}-W_{d}\right)\right),
\end{aligned}
$$

где $W_{d \leqslant \leqslant 2 d}$ обозначает траекторию винеровского процесса на отрезке $[d, 2 d]$. Чтобы получить искомый процесс на отрезке $[0, d]$, достаточно применить ту же процедуру, которую мы применяли для построения процесса $\left(\widehat{X}_{t}, \widehat{Y}_{t}\right)$ по процессу $\left(X_{t}^{0}, Y_{t}^{0}\right)$. Индуктивно продолжая указанную конструкцию, мы получим прогрессивно измеримый относительно потока $\left(\mathscr{F}_{t}^{W}\right)_{t \geqslant 0}$ процесс $\left(\widehat{X}_{t}, \widehat{Y}_{t}\right)$ на $[0, \infty)$, для которого

$$
\operatorname{Law}\left(\widehat{X}_{t}, \widehat{Y}_{t}, W_{t}\right)=\operatorname{Law}\left(\widetilde{X}_{t}, \widetilde{Y}_{t}, \widetilde{W}_{t}\right),
$$

следовательно, $\left(\widehat{X}_{t}, \widehat{Y}_{t}\right)$ и является искомым сильным решением уравнения (1). Так как для решений уравнения (1) выполняется единственность в смысле распределения, то, применяя теорему 3.2 из работы [4] и уже доказанное существование сильного решения, мы получаем, что для решений рассматриваемого уравнения выполняется потраекторная единственность.

Автор выражает благодарность В. И. Богачеву и А. Ю. Веретенникову за полезные обсуждения и замечания.

\section{СПИСОК ЛИТЕРАТУРЫ}

1. Abourashchi N., Veretennikov A. Yu. On stochastic averaging and mixing. - Theory Stoch. Process., 2010, v. 16(32), № 1, p. 111-129.

2. Campillo F., Pardoux E. Numerical methods in ergodic optimal stochastic control and application. - Lecture Notes in Control and Inform. Sci., 1992, v. 177, p. 59-73.

3. Campillo F. Optimal ergodic control for a class of nonlinear stochastic systems: application to semi-active vehicle suspensions. - Proceedings of the 28th IEEE Conference on Decision and Control (Tampa, 1989, New York: IEEE, 1989, p. 1190-1195). 
4. Черный A. С. О сильной и слабой единственности для стохастических дифференциальных уравнений. - Теория вероятн. и ее примен., 2001, т. 46, в. 3, с. 483-497.

5. Meyer-Brandis T., Proske F. Construction of strong solutions of SDE's via Malliavin calculus. - J. Funct. Anal., 2010, v. 258, № 11, p. 3922-3953.

6. Nualart D. The Malliavin calculus and related topics. Berlin: Springer-Verlag, 2006, $382 \mathrm{p}$.

7. Bogachev V. I. Gaussian Measures. Providence: Amer. Math. Soc., 1998, 433 p.

8. Bogachev V.I. Differentiable Measures and the Malliavin Calculus. Providence: Amer. Math. Soc., 2010, $488 \mathrm{p}$

9. Da Prato G., Malliavin P., Nualart D. Compact families of Wiener functionals. - C. R. Acad. Sci. Paris, 1992, v. 315, № 12, p. 1287-1291.

10. Бесов О.В., Ильин В. П., Никольский С. М. Интегральные представления функций и теоремы вложения. М.: Наука, 1975, 480 с.

11. Ikeda N., Watanabe S. Stochastic Differential Equations and Diffusion Processes. Amsterdam/Tokyo: North-Holland/Kodansha, 1989, 555 p.

12. Bally V., Saussereau B. A relative compactness criterion in Wiener-Sobolev spaces and application to semi-linear stochastic PDEs. - J. Funct. Anal., 2004, v. 210, № 2, p. $465-515$.

Поступила в редакцию 